\title{
Embedded stainless steel ring intrauterine device
}

The stainless steel ring intrauterine device (IUD) (Figure 1A) is widely used in China. ${ }^{1}$ Very often this type of IUD can be removed in the outpatient clinic by using an IUD removal hook.

A 32-year-old Chinese woman was referred for removal of an IUD that had been inserted 7 years previously in China. With the use of an IUD removal hook, only part of the device, which appeared to be a stainless steel ring, could be pulled out of the uterine cavity. It was then found to be adherent to the uterine wall, and with further traction the device was broken. Transvaginal pelvic sonography showed that an IUD fragment was embedded in the patient's right lateral uterine wall (Figure 2).

During hysteroscopy the end of the IUD fragment was barely seen covered by a thin layer of endometrium. By resecting the superficial myometrium more embedded IUD fragment was 


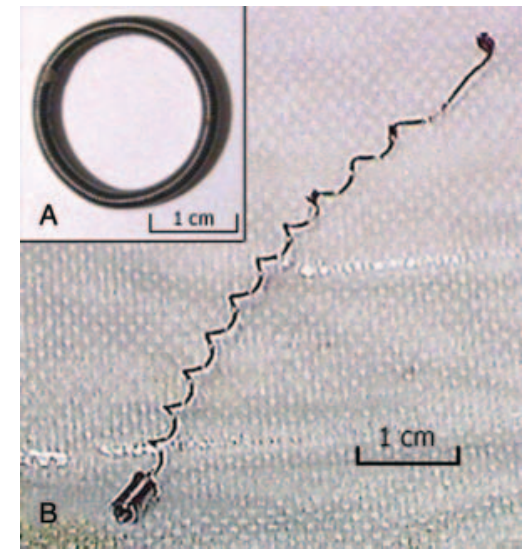

Figure 1 (A) The stainless steel ring intrauterine device (IUD). (B) The removed IUD fragment.

exposed. An attempt to manipulate the IUD fragment out of the myometrium using the resectoscope was unsuccessful and resulted in breakage of the electrode wire. The fragment was finally removed by pulling its end with a sponge forceps inserted into the uterine cavity (Figure 1B).

Given the popularity of worldwide travel nowadays, physicians are likely to see patients from all over the world, and therefore need to be familiar with this stainless steel ring IUD. Despite the ease of removing this type of IUD in most circumstances, difficulties can be encountered in occasional patients, as described here.

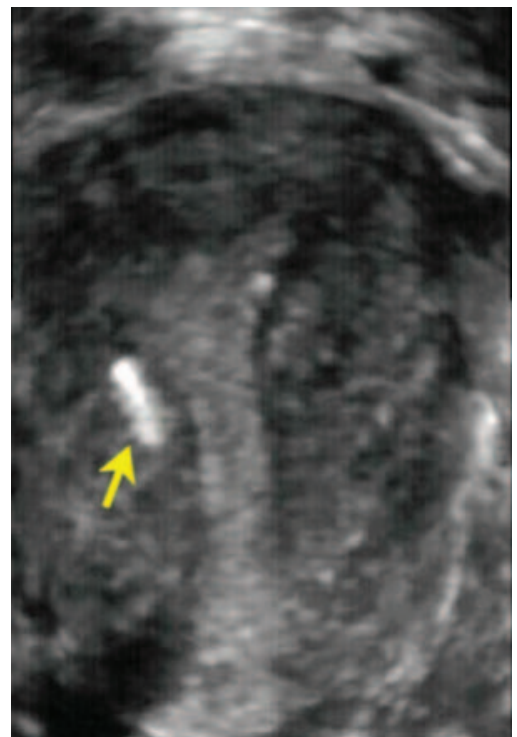

Figure 2 Transvaginal pelvic sonography, coronal view, showing the embedded intrauterine device fragment (arrow).

Vincent Y T Cheung, FRCOG, FRCSC

Consultant and Honorary Clinical Associate Professor, Department of Obstetrics and Gynaecology, Queen Mary Hospital, The University of Hong Kong, Hong Kong; vytc@hku.hk

\section{Competing interests None.}

Journal of Family Planning and Reproductive Health Care 2013;39:153-154.

doi:10.1136/jprhc-2013-100597

\section{REFERENCE}

1 Cheung VYT. A 10-year experience in removing Chinese intrauterine devices. Int J Gynecol Obstet 2010;109:219-222. 